\title{
Effectiveness of neurofeedback training on verbal memory, visual memory and self-efficacy in students
}

\author{
Mohammad Nazer ${ }^{1}$, Hanifeh Mirzaei ${ }^{2}$, Mohammadreza Mokhtaree ${ }^{3}$
}

${ }^{1}$ Assistant Professor, Psychiatry Department, Non-Communicable Diseases Research Center, Rafsanjan University of Medical Sciences, Rafsanjan, Iran

${ }^{2}$ M.Sc. of General Psychology, Islamic Azad University, Kerman Branch, Kerman, Iran

${ }^{3}$ M.Sc. of Educational Psychology, Social Determinations of Health Research Center, Rafsanjan University of Medical Sciences, Rafsanjan, Iran

\section{Type of article: Original}

\begin{abstract}
Background: Memory is the basis for the development of language skills and learning processes, and selfefficacy is one of the most important predictors of academic achievement.

Objective: This study aimed to determine the effects of neurofeedback training (NFB) on verbal and visual memory and self-efficacy in students of Rafsanjan University of Medical Sciences.

Methods: This quasi-experimental study was conducted on students of Rafsanjan University of Medical Sciences (Rafsanjan, Iran). Twenty-six students without severe neuropsychological disorder, history of epilepsy and drug abuse were selected randomly and divided into two groups. First, the participants completed the Morgan-Jinks Student Efficacy Scale (MJSES) and then were assessed by Kim Karad Visual Memory Test (KKVMT) and Wechsler Memory Scale for verbal memory (WMSVM). The experimental group underwent 15 sessions of neurofeedback training (NFB) for increase in sensorimotor response (SMR) in central zero (Cz) and frontocentral zero (FCz) location at the psychology clinic Rafsanjan University of Medical Sciences. Visual and verbal memory and self-efficacy were assessed by KKVMT, WMSVM and MJSES after completion of sessions and one month later. Posttest and follow-up of the control group were also carried out simultaneously with the experimental group. Data were analyzed in SPSS version 16, using descriptive statistical and repeated measures ANCOVA. The significance level of examining the hypotheses was set at $\mathrm{p} \leq 0.05$.

Results: The Mean \pm SD of verbal memory in the experimental group in pretest, posttest and follow-up was $20 \pm 1.9,22.58 \pm 2.1,22.41 \pm 2.06$ respectively $(\mathrm{p}<0.001$, Effect size $=0.53)$.

There were significant changes in short-term $(\mathrm{p}=0.001$, Effect size $=0.41)$ and long-term $(\mathrm{p}=0.001$, Effect size $=0.42$ ) visual memory. The changes of mid-term visual memory and self-efficacy in the experimental group were not significant $(\mathrm{p}=0.135, \mathrm{p}=0.062)$.

Conclusion: NFB is effective in improving verbal memory and some dimensions of visual memory. Further studies are needed to achieve better results.

Keywords: Neurofeedback, Memory, Self-efficacy
\end{abstract}

\section{Introduction}

Memory is a searchable storage system that has been studied more than anything else in recent years because of its very important role in learning and remembering (1). In addition to learning, memory also plays a fundamental role in an individual's thinking, creativity, planning and everyday behavior. Different structures in the brain control the stages of memory. During information storage, the brain's right hemisphere has the highest activity. According to the multi-store model of memory described by Atkinson and Shiffrin, the information processing normally begins with receiving environmental stimuli. The received information is transferred within less than a second to short-term memory. Otherwise, it will disappear. Vision is one of the main sensory channels through which a human receives

\section{Corresponding author:}

Hanifeh Mirzaei. Tel: +98.9134912219, Email: hanifeh.mirzaei@yahoo.com

Received: March 08, 2017, Accepted: December 26, 2017, Published: September 2018

iThenticate screening: October 07, 2017, English editing: August 20, 2018, Quality control: August 20, 2018

Ethics approval: $\mathrm{P} / \mathrm{D} / 31 / 5 / 3343$

(C) 2018 The Authors. This is an open access article under the terms of the Creative Commons Attribution-NonCommercialNoDerivs License, which permits use and distribution in any medium, provided the original work is properly cited, the use is non-commercial and no modifications or adaptations are made. 
and stores lots of information in his memory. Visual memory is the ability to store and retrieve experienced visual perceptions and emotions that can be recalled by environmental stimuli (2). Storage and retrieval of information heard or expressed by a person is also called verbal memory (3). Sometimes, some people have difficulties in visual or verbal memory despite having high intelligence and normal sensory channels. Incoming information is transferred to short-term memory through the senses and activation of the attention process (4). In addition, memory that plays a major role in learning inner psychological issues such as self-efficacy also plays an important role in this regard. Bandura proposed the concept of self-efficacy in 1997 as a missing element in learning theory. Bandura believes that self-efficacy is a determinant of behavior. Self-efficacy is also a cognitive process through which many social behaviors and personal characteristics are developed. Bandura proposed the theory about human reactiveness in which the role of self-beliefs is prominent in cognition, motivation and human behavior (5). Self-efficacy affects individuals' goals and actions and is affected by environmental conditions (6) such as recognition and acknowledgment from others, physical effects such as financial benefit, and self-evaluation (5). Also, in educational settings, self-efficacy refers to students' belief in their ability to perform certain educational tasks at specified levels (5). Thus, self-efficacy may increase as cognitive processes improve. Memory is affected by several factors and the stages of memory are controlled by different structures in the brain. Therefore, methods that can improve memory are of utmost importance. Biofeedback, an important modern method in the field of physiological psychology, is the process of recording biological information and returning it to the participant. Using a device attached to the body, this method provides information about the body's biological functions (7). Neurological feedback (NFB) is a widely used type of biofeedback that utilizes brainwaves, and is reported to improve memory (8). NFB is based on the brain's electrical activity (9) and involves training and learning self-regulation of brain activity (10). In NFB, the brain's electrical waves are received through electrodes connected to specific areas on the head, and are displayed in the form of shapes or moving images on the monitor, so that the participants can see their brainwaves. Then, with the help of an examiner, the participants begin to change their brainwaves by changing their thoughts and feelings. Since NFB is based on conditioning the participant (11), participants perceive the immediate changes occurring in their brainwaves in the form of changes in shapes and figures and hearing different sounds. Then, to receive a reward (point) again, participants begin to change their brainwaves by changing the direction of their thoughts and feelings. This process occurs regularly, and eventually, participants learn that they should change their brainwaves or thoughts in the direction intended by the examiner to receive a reward (12). This technique is used to achieve different objectives such as improving concentration and eliminating distractions and negative thoughts (13).

NFB training changes the brain activity and when skills are learned through this method, long-lasting changes are developed in the brainwave's structure (14). NFB training is used for enhancement of memory $(8,15-17)$. In addition, Walker et al., reported that the participants who could increase the sleep spindle activity by NFB, showed improvement in declarative memory performance. It should be noted that there is a relationship between sleep spindle generated by thalamocortical and verbal memory and learning (18). As mentioned before, self-efficacy believes in one's own abilities, and is highly correlated with cognitive functions, especially memory. Having a good memory increases believing in one's ability and success, and improves their performance $(19,20)$. Accordingly, the present study aimed to determine the effects of neurofeedback training on verbal and visual memory and selfefficacy in students of Rafsanjan University of Medical Sciences.

\section{Material and Methods}

\subsection{Trial design and participant}

This study was a quasi-experimental research with a control group that was conducted on the students of Rafsanjan University of Medical Sciences (Rafsanjan, Iran) in 2013. The participants were selected through simple random sampling based on a list of students obtained from the Department of Education. The followings were set as the exclusion criteria: students with 1) neurological disorders such as epilepsy and severe migraine headaches, 2) history of acute myocardial infarction.

\subsection{Intervention}

NFB training was held over a period of two months, three times a week. Participants sat on a chair in a quiet room, the electrodes were connected at central zero $(\mathrm{Cz})$, and fronto-central zero $(\mathrm{FCz})$ (based on 10-20 system). First, a wave base was recorded for 2 minutes. This wave base is the basis of the detection and evaluation of changes. After recording the wave base, the main part of the training program was started, which included recording and presenting brainwaves to the participant. The device displays the individual's different brainwaves on a computer monitor and simulates the waves as shapes and animations for better understanding. When the participants' brainwaves have reached the waves intended by the clinician (here an increase in the sensorimotor response (SMR) wave which is a 
part of beta waves with the frequency range from 12 to $15 \mathrm{~Hz}$ ), the shape or animation displayed will move further and the device will give a point to the individual based on the length of time that the individual can maintain the brainwaves intended by the clinician in the target frequency range. This process took 30-45 minutes per session.

\subsection{Research tools}

2.3.1. Kim Karad visual memory test

Kim Karad visual memory test was developed around 1945. In this test, some figures and shapes are displayed for the participants who are required to find them at a specific time among the other shapes provided. This test consists of a page containing 20 color images. The participant will look at the page for one minute. The participant must then place each piece in the right place in order to be scored, or he will not score if the piece is placed it in the wrong location. The range of scores is $0-20$ (21). The reliability of the instrument was assessed by test-retest method, with the sample size of 30, and its correlation coefficient with Andre Rey visual memory test was 0.68 .

2.3.2. Numerical - verbal memory tests

This test is a part of the Wechsler memory test. Numerical - verbal memory tests consist of four components including word-pair memory test, recognition memory, numerical memory and word-classification test; in the present study word-pair memory test and recognition memory subtest were used. This test consists of eight pairs of words. The examiner reads them to the participant. Then the examiner reads one word to the participant and he / she should tell another pair. This test could be repeated up to 8 times. The highest test score is 24 . The test reliability was assessed by test-retest method, with the sample size of 30, and reported as $80 \%$ for word-pair memory test and $73 \%$ for recognition memory, and the correlation coefficient of word memory test with the story recall test was 0.54 (22).

2.3.3. Morgan-Jinks self-efficacy scale

Morgan-Jinks self-efficacy scale has thirty items and three subscales: talent, effort and context. The items of this scale are scored with 4-point Likert scale. Ten items belong to the subscale of talent, ten items to the subscale of effort and ten items to the subscale of context. The range of this scale is 30-120. According to Morgan and Jinks, the reliability of the scale is 0.82 and the reliability of talent, effort and context subscales is $0.78,0.66$ and 0.70 , respectively (23). The reliability of the scale in this study was 0.71 .

\subsection{Sample size}

Based on the results of previous studies (24), the following formula and parameters were used to determine the sample size: $\mathrm{n}=\left(Z_{1-\alpha / 2}+Z_{1-\beta}\right)\left(S_{1}{ }^{2}+S_{2}{ }^{2}\right) /\left(\mu_{2}-\mu_{1}\right)^{2}$; where: $\mu_{1}=20, \mu_{2}=22.58, S_{1}=1.9, S_{2}=2.1, \alpha=0.05, \beta=20 \%$. The sample size was calculated as 12 according to the formula, but 15 participants were assigned to each group to ensure a sufficient sample size, and finally 12 participants remained in the experimental group and 14 in the control group. Four people did not complete the study.

\subsection{Statistical methods}

The data were analyzed by SPSS version 16 (SPSS Inc., Chicago, Illinois, USA) and statistical tests of repeated measures ANCOVA. The Shapiro-Wilk test was used to check the normality, which showed normal distribution for self-efficacy, but non-normal distribution for verbal memory of the participants $(\mathrm{p}=0.025)$.

\subsection{Ethical considerations}

This study protocol was approved at Islamic Azad University, Kerman Branch, Kerman, Iran (Ref: 134) and also approved by the Ethics Committee (Ref: P/D/31/5/3343). The selected participants agreed to participate in the study after the researcher presented the purpose and methods of the study to their respective faculties. Written informed consent was obtained from the participants. The researchers confirmed the confidentiality of the information of the participants using anonymous questionnaires.

\section{Results}

Thirty people enrolled in the study, but finally, twelve participants remained in the experimental group and fourteen remained in the control group (male: $26.9 \%$, female: $73.1 \%$ ) with the mean age of $22.42 \pm 1.75$ years. Figure 1 shows the study flow chart based on CONSORT 2010. All the participants were medical sciences students. The mean age of the experimental and control groups were $22.58 \pm 1.56$ and $22.28 \pm 1.93$ years respectively, which showed no statistically significant difference between the two groups ( $\mathrm{p}=0.45$ ). To assess the primary hypothesis of the present study, the descriptive statistics, including the mean and standard deviation of verbal and visual short-term, mediumterm and long-term memory and self-efficacy scores of the participants in both experimental and control groups, are shown in Table 1. The information in this table suggests changes in visual and verbal short-term memory scores in 
the posttest compared to the pretest. For inferential analysis of the results, analysis of variance with repeated measures and covariance were used. Therefore, it was necessary to investigate the normality of the data. Null hypothesis on the proportion of covariance of verbal memory variable with the unit matrix was rejected in all groups, but given that the probability level of Greenhouse-Geisser Correction was about zero and the size of both groups was equal, it is not necessary to observe the assumption (Molavi, 2005). However, the null hypothesis was confirmed for self-efficacy $(\mathrm{p}=0.063)$. The assumption of homogeneity of variances was assessed through Levene's test. The skewness of \pm 2 in the scores suggests normal distribution of the data (Table 2).

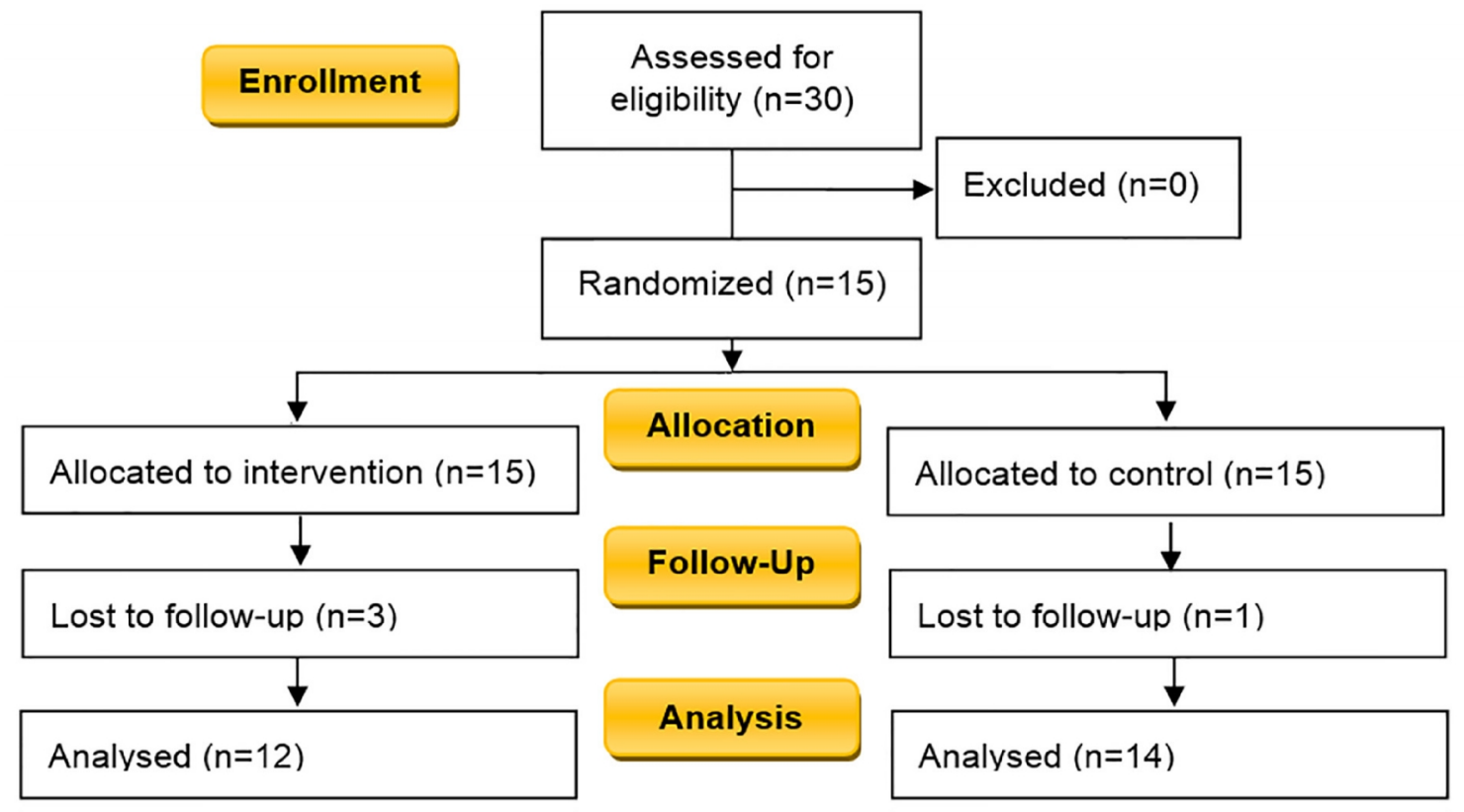

Figure 1. CONSORT 2010 Flow Diagram of the study

Table 1. Descriptive indicators of verbal memory, visual memory and self-efficacy

\begin{tabular}{|c|c|c|c|c|c|c|c|c|c|}
\hline \multirow{2}{*}{\multicolumn{2}{|c|}{ Variables }} & \multicolumn{4}{|c|}{ Experimental group (Mean \pm SD) } & \multicolumn{4}{|c|}{ Control group (Mean $\pm \mathrm{SD})$} \\
\hline & & Pretest & Posttest & Follow-up & p- value & Pretest & Posttest & Follow-up & p-value \\
\hline \multicolumn{2}{|c|}{ Verbal memory } & $20 \pm 1.9$ & $22.58 \pm 2.1$ & $2.41 \pm 2.06$ & 0.001 & $1.78 \pm 2.77$ & $21.85 \pm 2.93$ & $22 \pm 2.45$ & 0.57 \\
\hline \multirow{3}{*}{$\begin{array}{l}\text { Visual } \\
\text { memory }\end{array}$} & Short-term & $1.58 \pm 0.52$ & $2.41 \pm 0.66$ & $2.5 \pm 0.52$ & 0.001 & $1.93 \pm 0.83$ & $2 \pm 0.96$ & $1.93 \pm 0.83$ & 0.73 \\
\hline & Medium-term & $1.83 \pm 0.39$ & $2 \pm 0.6$ & $2.16 \pm 0.39$ & 0.13 & $1.93 \pm 0.61$ & $1.93 \pm 0.82$ & $2 \pm 0.68$ & 0.77 \\
\hline & Long-term & $10.8 \pm 0.29$ & $2.08 \pm 0.28$ & $2.16 \pm 0.39$ & 0.001 & $2.07 \pm 0.61$ & $2.21 \pm 0.58$ & $2.07 \pm 0.61$ & 0.57 \\
\hline \multicolumn{2}{|c|}{ Self-efficacy } & $59 \pm 5.42$ & $59.75 \pm 5.8$ & $9.25 \pm 5.72$ & 0.062 & $58.93 \pm 4.99$ & $59 \pm 4.99$ & $58.85 \pm 5$ & 0.093 \\
\hline
\end{tabular}

Table 2. The p-value of normality of distribution, normality of data, homogeneity of variance and test for sphericity

\begin{tabular}{|l|l|l|l|l|l|l|}
\hline Source & Skewness & Kurtosis & Shapiro-Wilk & Levene's test & Mauchly's W \\
\hline Verbal Memory & & -0.304 & -1.102 & 0.025 & 0.098 & 0.132 \\
\hline \multirow{3}{*}{ Visual memory } & Short-term & 0.369 & -0.857 & 0.001 & 0.204 & 0.091 \\
\cline { 2 - 7 } & Mid-term & -0.207 & 1.027 & 0.001 & 0.428 & 0.015 \\
\cline { 2 - 7 } & Long-term & 0.703 & -0.575 & 0.001 & 0.108 & 0.001 \\
\hline Self-efficacy & -0.299 & -0.283 & 0.119 & 0.574 & 0.437 \\
\hline
\end{tabular}

Repeated measures ANCOVA was used to compare the difference of the mean scores of the research variables. The results showed that the interaction between measurement stages and group membership in verbal and visual shortterm and long-term memory was significant $(\mathrm{p}<0.001, \mathrm{p}=0.001, \mathrm{p}=0.001)$; i.e., the difference between the mean of scores of the variables was significant in pretest, posttest and follow-up stages (Table 3 ). Analysis of covariance was used to investigate whether this change in scores was due to NFB or pretest. After adjusting pretest scores, there was a significant effect of the factor among the participants for verbal memory (effect size $=0.53, p=0.00, F 23.1=26.21$ ) and visual short-term memory (effect size $=0.61, p=0.00, F 23.1=36.03$ ). Analysis of the results showed that NFB 
could not develop a significant change in visual medium-term memory $(\mathrm{p}=0.57)$, visual long-term memory $(\mathrm{p}=0.55)$ and self-efficacy of the experimental group $(\mathrm{p}=0.094)$ (Table 4).

Table 3. The interaction effect between group membership and repeated measures of verbal memory, visual memory and self-efficacy subjects

\begin{tabular}{|l|l|l|l|l|l|l|l|l|l|}
\hline Source & \multicolumn{2}{l|}{} & SS & df & MS & F & p-value & ES & Power \\
\hline Interactions & Verbal memory & 22.59 & 2 & 11.29 & 30 & $<0.001$ & 0.55 & 1 \\
\cline { 2 - 11 } & Visual memory & Short-term & 3.11 & 2 & 1.56 & 16.77 & 0.001 & 0.41 & 0.99 \\
\cline { 3 - 11 } & & Medium-term & 0.23 & 1.53 & 0.15 & 0.68 & 0.47 & 0.028 & 0.14 \\
\cline { 2 - 10 } & Long-term & 4.22 & 1.17 & 3.62 & 17.6 & 0.001 & 0.42 & 0.99 \\
\cline { 2 - 9 } & Self-efficacy & & 1.2 & 1.87 & 0.65 & 1.99 & 0.15 & 0.076 & 0.39 \\
\hline
\end{tabular}

SS: Sum of Square, df: Degree of Freedom, MS: mean of Square, ES; Effect size

Table 4. Summary results of analysis of covariance (ANCOVA) for the effectiveness of NFB on verbal memory, visual memory and self-efficacy of the experimental group, with covariate of pretest

\begin{tabular}{|l|l|l|l|l|l|l|l|l|l|}
\hline Variable & SC & SS & df & MS & F & p-value & ES & Power \\
\hline Verbal memory & Pretest & 116.73 & 1 & 116.73 & 111 & $<0.00$ & 0.83 & 1 \\
\cline { 3 - 11 } & Group & 27.56 & 1 & 27.56 & 26.21 & $<0.00$ & 0.53 & 0.99 \\
\hline \multirow{3}{*}{ Visual memory } & \multirow{2}{*}{ Short-term } & Pretest & 14.45 & 1 & 14.45 & 134.79 & 0.002 & 0.85 & 1 \\
\cline { 3 - 11 } & & Group & 3.86 & 1 & 3.86 & 36.03 & 0.001 & 0.61 & 1 \\
\cline { 3 - 11 } & \multirow{2}{*}{ Medium-term } & Pretest & 3.68 & 1 & 3.68 & 9.16 & 0.006 & 0.28 & 0.82 \\
\cline { 3 - 10 } & \multirow{2}{*}{ Long-term } & Group & 0.13 & 1 & 0.13 & 0.32 & 0.57 & 0.014 & 0.085 \\
\hline & & Pretest & 0.49 & 1 & 0.49 & 2.38 & 0.13 & 0.094 & 0.31 \\
\cline { 3 - 10 } & Group & 0.076 & 1 & 0.076 & 0.37 & 0.55 & 0.016 & 0.09 \\
\hline \multirow{3}{*}{ Self-efficacy } & Pretest & 499.55 & 1 & 449.55 & 640.25 & $<0.00$ & 0.96 & 1 \\
\cline { 3 - 10 } & & Group & 2.38 & 1 & 2.38 & 3.06 & 0.094 & 0.12 & 0.39 \\
\hline
\end{tabular}

$\mathrm{P}<0.05$, SC: Source of changes, SS: Sum of Square, df: Degree of Freedom, MS: mean of Square, ES: Effect size

\section{Discussion}

The aim of this study was to determine the effects of NFB training on verbal and visual memory and self-efficacy in students of Rafsanjan University of Medical Sciences. The results of this study showed a significant difference between the experimental and control groups in terms of verbal memory performance, that is, the experimental group had a better performance on the posttest. Verbal memory is one of the important cognitions in the correct naming of emotional facial expression recognition. Correct encoding of the information about the recognition of facial expressions of excitement depends on verbal memory (25). The NFB improves the activity of the frontal lobe as well as the activation of the neural circuits associated with actuator functions and memory and improves the defects of executive function and active memory (26). The findings of the present study are consistent with the results of Alidusti et al. and Gholizad et al. $(24,27)$. Also, Sarvghad et al. demonstrated that the NFB led to an increase in the verbal memory in women after breast cancer treatment (28).

In this study, increased SMR protocol was used in $\mathrm{Cz}$, where sensorimotor, motor and cingulate cortices are located. Regarding the relationship between memory and sensorimotor cortex of the brain, it seems that brainwave changes in the zone will lead to changes in the performance of various functions in this zone such as memory. In the present study, NFB had no effect on visual medium- and long-term memory. The results of this study contrasted with the study of Gholizadeh et al. (24). It seems that the visual memory is related with various variables, such as intelligence (29-31), critical thinking (32) and motivation (33) that are not assessed in this study. Since the encoding zone, verbal memory and working verbal memory are on the left side of the brain and visual-spatial memory is on the right side of the brain, and given that the reference electrode was placed on the left side of the head in this study, and as a result, the left side of the brain was further under beta waves, perhaps it was more effective on verbal memory and was less effective on visual memory. In addition, Fatollahpour et al. studied the effectiveness of NFB on intelligence in children with dyslexia. In their study, long-term memory significantly changed after NFB (34). Perhaps the reason for this inconsistency is the protocol performed. The protocol used in this study was increased SMR and reduced theta in the $\mathrm{Cz}$, while the researchers mentioned increased SMR, decreased theta from 4 to $7 \mathrm{~Hz}$ and beta from 22 to $30 \mathrm{~Hz}$ in $\mathrm{C} 3$ and $\mathrm{C} 4$ zones in addition to increase SMR and decrease theta in the Cz. In the present study, NFB could not increase self-efficacy of the participants in the experimental group. Self-efficacy is an internal and psychological 
variable affected by many variables. It needs time, and the person should be placed in different practical situations to try his skills and turn them into beliefs over time. Since the measurement was performed at posttest and follow-up immediately, and one month after NFB, perhaps it was too short for skills to change to beliefs and thus increase selfefficacy.

\section{Conclusions}

It can be concluded that NFB is effective in improving verbal memory and some dimensions of visual memory. Therefore, NFB can be used to improve the memory of individuals in various parts of life and work. It is suggested that the impact of NFB on academic achievement be assessed through improved work memory. Further studies are needed to clarify the effectiveness of NFB training in different psychological variables or physical disorders.

\section{Acknowledgments:}

This article was extracted from Ms. Hanifeh Mirzai's dissertation in M.Sc. of Psychology. The authors express their thanks and appreciations to the Deputy of Research of Islamic Azad University, Kerman branch (Kerman, Iran) and research center of Rafsanjan Moradi hospital (Rafsanjan, Iran). We would like to convey thanks to everyone who helped us with this study and special thanks to the students who contributed to our study.

\section{Conflict of Interest:}

There is no conflict of interest to be declared.

Authors' contributions:

All authors contributed to this project and article equally. All authors read and approved the final manuscript.

\section{References:}

1) Kandel RE, James H, Thomas MJ. Principles of Neural Science. New York MCgraw-Hill Companies. 2000.

2) Pakatchi R, Yaryari F, Moradi A. The Effects of Teaching via Computer Games on the Performance of Visual Memory for the Dyslexic Students. Journal of Exceptional Education. 2013; 6(119): 30-8.

3) Miotto EC, Balardin JB, Savage CR, Martin Mda G, Batistuzzo MC, Amaro Junior E, et al. Brain regions supporting verbal memory improvement in healthy older subjects. Arq Neuropsiquiatr. 2014; 72(9): 66370. PMID: 25252229.

4) Solso R, MacLin OT, MacLin MK. Cognitive Psychology. 8th ed: Pearson; 2007.

5) Bandura A. Social cognitive theory: an agentic perspective. Annu Rev Psychol. 2001; 52: 1-26. doi: 10.1146/annurev.psych.52.1.1. PMID: 11148297.

6) Schunk DH. Goal Setting and Self-Efficacy During Self-Regulated Learning. Educational Psychologist. 1990; 25(1): 71-86. doi: 10.1207/s15326985ep2501_6.

7) Nosratabadi M. Examining the application of QEEG in neurofeedback diagnosis for treatment of ADHD. Tehran: Allameh Tabataba'i University, Iran. 2006.

8) Wang JR, Hsieh S. Neurofeedback training improves attention and working memory performance. Clinical neurophysiology: official journal of the International Federation of Clinical Neurophysiology. 2013; 124(12): 2406-20. doi: 10.1016/j.clinph.2013.05.020. PMID: 23827814.

9) Datko M, Pineda J, Muller RA. Positive effects of neurofeedback on autism symptoms correlate with brain activation during imitation and observation. The European journal of neuroscience. 2017.

10) Gunkelman JD, Johnstone J. Neurofeedback and the Brain. Journal of Adult Development. 2005; 12(2): 93 8. doi: 10.1007/s10804-005-7024-x.

11) Gruzelier JH, Egner T, Valentine E, Williamon A. EComparing learned EEG self-regulation and the Alexander technique as means of enhancing musical performance. 7 th international conference on music perception and cognition. Sydne, Adelaide 2002.

12) Demos JM. Getting started with Neurofeedback. US: W. W. Norton \& Company. 2005.

13) Luctkar-Flude M, Groll D. A Systematic Review of the Safety and Effect of Neurofeedback on Fatigue and Cognition. Integrative cancer therapies. 2015; 14(4): 318-40. doi: 10.1177/1534735415572886. PMID: 25716351.

14) Monastra VJ, Monastra DM, George S. The effects of stimulant therapy, EEG biofeedback, and parenting style on the primary symptoms of attention-deficit/hyperactivity disorder. Applied psychophysiology and biofeedback. 2002; 27(4): 231-49. PMID: 12557451. 
15) Escolano C, Aguilar M, Minguez J. EEG-based upper alpha neurofeedback training improves working memory performance. Conf Proc IEEE Eng Med Biol Soc. 2011; 2011: 2327-30. doi: 10.1109/IEMBS.2011.6090651. PMID: 22254807.

16) Xiong S, Cheng C, Wu X, Guo X, Yao L, Zhang J. Working memory training using EEG neurofeedback in normal young adults. Bio-medical materials and engineering. 2014; 24(6): 3637-44. doi: 10.3233/BME141191. PMID: 25227078.

17) YuLeung To E, Abbott K, Foster DS, Helmer D. Working Memory and Neurofeedback. Applied neuropsychology Child. 2016; 5(3): 214-22. doi: 10.1080/21622965.2016.1167500. PMID: 27191218.

18) Walker MP, Brakefield T, Morgan A, Hobson JA, Stickgold R. Practice with sleep makes perfect: sleepdependent motor skill learning. Neuron. 2002; 35(1): 205-11. PMID: 12123620.

19) Beaudoin M, Desrichard O. Are memory self-efficacy and memory performance related? A meta-analysis. Psychological bulletin. 2011; 137(2): 211-41. doi: 10.1037/a0022106. PMID: 21244133.

20) Hastings EC, West RL. Goal orientation and self-efficacy in relation to memory in adulthood. Neuropsychol Dev Cogn B Aging Neuropsychol Cogn. 2011; 18(4): 471-93. doi: 10.1080/13825585.2011.575926. PMID: 21728891, PMCID: PMC3132153.

21) Garmabi M, Adib-Sereshki N, Taheri M, Movallali G, Seyyed Noori SZ. The Effectiveness of Visual Perception Skills Training on Short-Term Visual Memory of Children with Hearing Impairment. Quarterly Journal of Child Mental Health. 2016; 3(1): 71-80.

22) Kajbaf MB, Lahijanian Z, Abedi A. A Comparison of Memory Profile in Normal Children and Children with Learning Disabilities in Dictation, Mathematics and Reading. Advances in Cognitive Science. 2010; 12(1): 17-5.

23) Jinks J, Morgan V. Children's Perceived Academic Self-Efficacy: An Inventory Scale. The Clearing House. 1999; 72(4): 224-30. doi: 10.1080/00098659909599398.

24) Gholizadeh Z, BabapourKheirodin J, Rostami R, Beyrami M, Poursharifi H. Effectiveness of neurofeedback on working memory. Journal of Psychology of University of Tabriz. 2009; 5(18): 87-100.

25) Phillips LH, Channon S, Tunstall M, Hedenstrom A, Lyons K. The role of working memory in decoding emotions. Emotion. 2008; 8(2): 184-91. doi: 10.1037/1528-3542.8.2.184. PMID: 18410192.

26) Heydarinasab L, Madani AS, Yaghoubi H, Rostami R, Kazemi R. The effectiveness of neurofeedback with computrized training in improving working memory in adults with attention deficit disorder/ hyperactivity. scientific magazine yafte. 2016; 18(1): 101-12.

27) Alidusti Shahraki N, Asgari K. The Effectiveness of neurofeedback training on improvement of Working Memory students with Dyslexia: A single case study. Journal of Shahrekord Uuniversity of Medical Sciences. 2016; 18(1): 105-21.

28) Sarvghadi P, Angooti Oshnari L, Mirzakhay N, Akbarfahimi M, Rezaei M, Akbarzade Baghban A. The effects of neurofeedback on verbal memory in women after breast cancer treatment. The Scientific Journal of Rehabilitation Medicine. 2016. PMCID: PMC4898843.

29) Abedini Mazraeh S. Comparison of Active Memory Functions (Visual-Audio) in Normal and Short-lived Children. Tehran: Islamic Azad University, Tehran branch. 2012.

30) Tajabadipour S, Sayed Mirzaee SZ. Relationship of the theory of mind with visual and auditory memory in students with learning disabilities. JOIH. 2016; 18(1): 725-37.

31) Zare H, Ali-Pour A, Nazer M, Mokhtari MR. Study of Verbal and Visual Memory in Patients with Schizophrenia Diagnosed According to the Prognosis and the Level of General Intelligence. Archives of Rehabilitation. 2012; 13(3): 88-94.

32) Amiri M, Pourmoradkohan P, Farhadi nia H, Shahgholian M. The relationship between visual-spatial memory with critical thinking disposition and attitude toward creativity Given the preference hemisphere, Basis for the generalizability of the right hemisphere cognitive functions. Journal of Cognitive Psychology. 2017; 4(4): 31-40.

33) Sanada M, Ikeda K, Kimura K, Hasegawa T. Motivation enhances visual working memory capacity through the modulation of central cognitive processes. Psychophysiology. 2013; 50(9): 864-71. doi: 10.1111/psyp.12077. PMID: 23834356.

34) Fatholahpour L, BabapourKheirodin J, Mahdavian H, BafandeGharamaleki H. Comparison of the effectiveness of neurofeedback and Fernald's multisensory approach on the intelligence of children diagnosed with Dyslexia disorder. Learning disabilities. 2013; 2(4): 103-23. 